


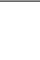




\title{
La educación virtual como favorecedora del aprendizaje autónomo ${ }^{1}$
}

Fecha de recepción: 26 de julio de 2011

Fecha de aprobación: 2 de septiembre de 2011

\section{César Augusto Sierra Varón}

Politécnico Grancolombiano

csierrav@poligran.edu.co

Psicólogo. Pontificia Universidad Javeriana. Integrante del grupo de investigación

"Comportamiento, cognición y neurociencias", bajo la línea de investigación "Responsabilidad social universitaria.

\author{
Fecha de aprobación: 2 de septiembre de 2011
}

\section{Resumen}

Son varios los aspectos que han venido evolucionando a lo largo del tiempo en relación a prácticas pedagógicas y la manera de entender la relación entre enseñanza y aprendizaje. Así mismo, en la actualidad se pretende cambiar el énfasis de la pedagogía centrada en la enseñanza a una pedagogía centrada en el aprendizaje, para que de esta manera los estudiantes adopten una posición más activa frente a sus propios procesos de aprendizaje y se favorezca el aprendizaje autónomo, buscando que los estudiantes aprendan a aprender. En el presente artículo se desarrollan los temas que defienden la tesis que afirma que la modalidad de educación virtual favorece el desarrollo del aprendizaje autónomo en los estudiantes, gracias al apoyo de las TIC (Tecnologías de la Información y las Comunicaciones) y a ciertas características que deben poseer o desarrollar los nuevos aprendices. Desde la revisión teórica llevada a cabo en la presente investigación, se exponen aspectos que orientan a los docentes de educación virtual a tener en cuenta algunas características importantes en las modalidades de educación virtual, para que las asuman como pertinentes a la hora de desarrollar sus cursos o módulos en esta modalidad. No se trata de dejar solos a los estudiantes, sino de desarrollar estrategias que favorezcan

\begin{abstract}
There are several aspects that have evolved throughout time regarding educational practices and the understanding of the relationship between teaching and learning. Likewise, currently, the emphasis on the pedagogy centered on teaching is to be changed by a pedagogy centered on learning. This way, students adopt an active position towards their own learning processes, and autonomous learning is fostered because students learn to learn. This article develops those subjects that support the thesis which states that virtual education supports the development of autonomous learning among students due to the use of IT and certain characteristics new learners should have or develop. From the theoretical revision carried out in this investigation, we show several aspects that guide teachers of virtual education in order to keep into account certain important characteristics of this type of education when developing their classes or modules. We do not suggest that students are to be left alone, but we recommend that teachers develop strategies that promote autonomy within the learning process being carried out. At the same time, we clarify that paradigm changes regarding education should be changed from teachingcentered pedagogies to learning-centered ones, enabling students to have a more active role.
\end{abstract}


la autonomía en el proceso de aprendizaje que llevan a cabo. Al mismo tiempo, se aclara que los cambios de paradigma en materia de educación deben pasar de las pedagogías centradas en la enseñanza, para pasar a pedagogías centradas en el aprendizaje, dando lugar a un rol más activo de los estudiantes.

\section{Palabras clave}

Aprendizaje autónomo, educación virtual, pedagogía, enseñanza, aprendizaje.

\section{Keywords}

Autonomous Learning, Virtual Education, Pedagogy, Teaching, Learning. 
Muchos aspectos de la humanidad tienen que ver con su propia evolución, siendo aspectos que se transforman para el mejoramiento de la calidad de vida de las personas. Un aspecto importante en la formación de toda persona es la educación.

Haciendo un rápido recorrido por las herramientas que hemos utilizado para educar, se puede iniciar con la expresión oral por medio de relatos de cada una de las culturas, continuando con el lenguaje pictórico en el cual se plasmaban tradiciones, conocimientos, etc. Después, con el lenguaje escrito, el hombre pudo enseñar y educar, y con el invento de la imprenta, por medio de textos se amplió la cobertura para transmitir el conocimiento a otros a largas distancias. La pedagogía se basó, en todo este gran avance que hemos tenido a través del tiempo, en la idea de que las personas debían ser educadas y alfabetizadas.

Para la educación se han utilizado herramientas incluyendo la tablilla, el pizarrón, la tiza, los cuadernos, el tablero acrílico, etc., y poco a poco estos elementos se han ido eliminando gracias al desarrollo tecnológico. Con el invento de los computadores, se ha logrado desmontar los espacios y objetos físicos para implementar los espacios y los objetos virtuales. Los cambios y avances tecnológicos han llevado a que en el mundo se presenten momentos de creciente complejidad e incertidumbre debido a que las condiciones del entorno varian a la velocidad que el cambio impone.

De esta manera, las Tecnologías de la Información y la Comunicación (TIC) están produciendo unos cambios que probablemente hace algunos años ni siquiera se sospechaban (Rincón y Romero, 2006), y tales tecnologías a su vez asumen un rol protagónico en la evolución del conocimiento de los últimos años (Pirela De Odón, 2004) mejorando las prácticas educativas en la educación actual (Fernández y Córdoba, 2006) y proporcionando una alternativa más para complementar los contenidos de aprendizaje de las nuevas modalidades educativas (Rada, 2006). En los últimos años, este rápido avance de las Tecnologías de la Información y la Comunicación han permeado los múltiples escenarios y formas de abordar los procesos de enseñanza-aprendizaje en educación superior (Serrano y Narváez, 2010).
En la actualidad, ya no hay impedimento de las distancias para la educación; ya no es necesario asistir a salones, sino que desde el lugar donde nos encontremos podemos acceder a estudios de formación académica por medio de la educación virtual. Imbernón, Silva y Guzmán, (2011), citando a Uceda y Senén (2010), refieren que una de las características que más se resaltan dentro del proceso de la virtualización del proceso de enseñanzaaprendizaje es el uso de equipos informáticos mediante la herramienta de Internet, siendo ésta una forma emergente de proporcionar conocimientos y educación a amplios sectores de la población (Páez y Arreaza, 2005).

Esta es la manera como la ciencia y el conocimiento evolucionan, aunque con ello se presenten problemas de incertidumbre, proble-matización, etc. Las críticas a modelos vigentes no siempre son bienvenidas (González, 2006). Sin embargo, es necesario tener en cuenta que los modelos de formación, de educación y de aprendizaje a nivel universitario, también se ven afectados por tal evolución, lo cual lleva a cambios. Tales cambios llevan a una modificación de la actual formación en cuanto a educación superior se refiere, apuntando hacia una idea de cambio profundo en las universidades mediante un conjunto de propuestas que lo justifican, llegando a un nuevo paradigma donde el eje de su discurso radica en el aprendizaje (Rué, 2009). Como ejemplos se pueden mencionar el aprendizaje basado en problemas, fundamentado en la teoría constructivista (Gorbaneff y Cancino, 2009), o el método de casos de estudio, "estrategia didáctica que permite contextualizar situaciones y problemas del mundo real para guiar el aprendizaje de los estudiantes" (Monguet, Fábregas, Delgado, Grimón \& Herrera, 2006).

Independiente de las épocas, sucesos y personajes que puedan servir de referencia para situar los inicios de la educación virtual, se encuentra indiscutiblemente el crecimiento y desarrollo de esta modalidad de educación. Y como cualquier proyecto pedagógico, la modalidad de educación virtual es innovadora e implica cambios en diferentes órdenes que son complementarios.

En los últimos años y especialmente a partir de la segunda mitad del siglo XX, se han presentado variaciones sustanciales en las 
concepciones, enfoques y metodologias sobre educación y aprendizaje, pero a pesar de estas variaciones el estudiante siempre ha dependido del profesor. Con la educación virtual y el apoyo de las herramientas tecnológicas, se pone a disposición de los estudiantes una gran gama de recursos que hacen que el aprendizaje se convierta en algo dinámico e interactivo, llegando a ser más significativo (Imbernón et al., 2011). Las plataformas electrónicas son la herramienta mediante la cual se pueden llevar a cabo actividades como las evaluaciones, las asesorias, las entregas de trabajos, foros virtuales (Software ForChat) (Varini y Axt, 2008), chats, entre otras (Torres y Benitez, s.f.).

La modalidad de educación virtual asume el cambio educativo, atendiendo a los múltiples factores involucrados en el desarrollo de esta modalidad. Desde esta perspectiva se pueden establecer directrices claras y viables para poder encauzar las acciones de acuerdo con los fines de la filosofia y politica educativas que las instituciones mismas plantean (Enriquez, 2001).

A pesar de que los tiempos y el sistema cambien, el objetivo importante dentro del proceso de la educación, sea presencial o virtual, es el estudiante. Asi lo refiere Rosalba Rodríguez (2009) en su documento "Metodología del trabajo académico" al decir que "Históricamente el centro de la educación virtual lo ocupa el estudiante, sujeto activo y responsable, capaz de encargarse autónomamente de su propio aprendizaje, empleando para ello diversas estrategias, desarrolladas con el apoyo de diferentes mediaciones y medios pedagógicos (pg. 50)Al observar y analizar lo que Badía y Monimó (2001) plantean en relación a la educación virtual, que con la ayuda de los avances tecnológicos ha tenido en los últimos años un encuentro muy similar al que presenta un artista en su máximo nivel de estrellato, se debe aclarar si la educación virtual se trata de un simple fenómeno de moda pasajero o si suple las verdaderas necesidades que encuentra el mundo de la educación. Esto, teniendo en cuenta que aún nos falta perspectiva y experiencia práctica en este campo para valorar su trayecto en el avance del campo de la educación.

La educación virtual se puede encontrar como un campo apropiado para poder analizar y comprender los desafios que impone la cultura actual en relación a sus entidades educativas frente a la sociedad de la información y el conocimiento. La aparición del ciberespacio y lo virtual son dos aspectos que han inducido al estudio de estos fenómenos con el objetivo fundamental de analizar la conveniencia de la teoria que subyace a la educación virtual junto con la teoría que se gesta para ser aplicada en entornos virtuales de aprendizaje (Garduño, 2005).

Un factor de mucha influencia en la educación a distancia son las TIC; el aspecto esencial de éstas es que no hay interacción presencial directa entre docente y alumno. Esto no quiere decir que ya no exista comunicación entre docente (tutor) y alumnos, sino que esta comunicación no presencial se realiza a través de los medios de comunicación (aprendizaje diferente), en el cual el estudiante desarrolla con mayor énfasis su autonomia, su autorregulación, su disciplina y sus hábitos de estudio, ya que es él mismo quien se encarga de sacar el mayor provecho a su proceso de formación académica (Ferroni y Velásquez, 2005). Los procesos de educación y aprendizaje virtual hacen uso de herramientas tutoriales para explotar los procesos de aprendizaje tanto individuales como grupales (Garduño, 2009).

La educación virtual y las nuevas tecnologias de la información y la comunicación van de la mano, por ello es importante resaltar algunas ventajas que plantean Rodríguez, Hernández y Albarracín (2008):

Se facilita la comunicación entre profesores (tutores) y alumnos, eludiendo los problemas de horarios y distancias.

Se facilitan nuevos canales de comunicación entre los estudiantes, según sus intereses e inquietudes.

Se suministra una cantidad enorme de información, con gran rapidez y a un bajo costo.

Sin embargo, la educación virtual no ha tenido la valoración y el respeto que merece y padece de cierto descrédito, pero también es altamente valorada por otros colectivos y por el contexto social en el que vivimos (Badia y Monimó, 2001). Rogers (1995), citado por López (2005), plantea que es muy dificil hacer que una nueva idea sea adoptada a pesar de que tenga grandes y obvias ventajas, llevando a que muchas innovaciones tomen un largo periodo de tiempo para que puedan ser ampliamente aceptadas. 
Así mismo, la educación virtual pretende constituirse en una opción que sea capaz de multiplicar y diversificar la educación para los individuos y grupos sociales sin tener que distinguir edad, raza y ubicación geográfica y que se encuentre basada en la democratización y la equidad. Ello se puede equiparar a la expresión: "la educación en la comunidad y la comunidad en la educación" que refiere al nuevo reto de desarrollar una educación abierta al entorno, equitativa e inclusiva (Merino, 2009). Tal alternativa se debe también a los nuevos retos que enfrenta la educación superior como por ejemplo la alta demanda y el poco espacio físico disponible. Por esta razón las nuevas tecnologias se convierten en una herramienta para ofrecer nuevas formas de intervención para atender las necesidades educativas (Iriarte, 2010).

En la modalidad de educación virtual, la Academia es la que se desplaza y llega a los hogares, a sitios de trabajo o a centros de estudio. Tales prácticas educativas se iniciaron con el objetivo de hacer llegar la educación a todo aquel que la necesita (Gutiérrez, Hernández, González, Guevara \& Flórez, 2004).

Aunque la educación virtual presenta ciertas caracteristicas que la distinguen y la hacen diferente en algunos aspectos de otros tipos de enseñanza y aprendizaje, tales rasgos pueden ser lo suficientemente diferenciales como para que emerja un nuevo paradigma en educación (Badía y Monimó, 2001). Si se acepta que la educación virtual es un contexto más donde se pueden desarrollar los procesos de enseñanza y aprendizaje, se deben interpretar los mismo criterios psicopedagógicos aplicables a la modalidad de educación presencial.

De igual forma, la Universidad tiene que cumplir un papel protagónico en la sociedad y asi lo ha demostrado a lo largo de su historia. En su labor de actualización por las nuevas y mejores modalidades de educación, buscan un modelo pedagógico nuevo y alterno que da el paso de la cultura de enseñar a la cultura de aprender (González, 2006).

Un aspecto fundamental para analizar en el proceso de educación virtual es el desarrollo del aprendizaje autónomo. ¿Será que en realidad somos independientes cuando estudiamos? Si se toma el término independencia por la definición que nos ofrece el diccionario, encontramos que es "la no admisión de control o dependencia externa" (Diccionario de la Real Academia de la Lengua, 2001). De esta forma, parece evidente que no se podría hablar de estudio independiente dado que continuamente nos acompañan factores externos como las instrucciones de nuestros tutores sobre lo que se debe realizar en las asignaturas y los textos digitalizados. También nos refieren el nivel de exigencia sobre lo que se debe realizar, incluso algunas sugerencias sobre cómo se deberian realizar las actividades (ensayos, mapas conceptuales, artículos, etc.) (Badia, 2001).

Se acaba de citar un concepto clave en la noción de autonomía de aprendizaje, ya que se trabaja el concepto de autonomía no como independencia, sino como "la facultad de tomar decisiones que permitan regular el propio aprendizaje para aproximarlo a una determinada meta en el seno de unas condiciones específicas que forman el contexto de aprendizaje" (Badia, p.12. 2001).

Tal facultad de poder gobernarse a sí mismo cuando se aprende, se puede dar gracias a la capacidad humana de saber cuándo una información ya existe en nuestra memoria o es completamente nueva. Tal capacidad recibe el nombre de metacognición y puede entenderse como la competencia que nos permite a los seres humanos ser conscientes de los procesos y productos que elaboramos en nuestra mente.

Por otro lado, la educación virtual no puede ofrecer sus ventajas por si sola, ya que depende además de ciertos factores que influyen en el éxito de esta experiencia de formación autónoma. Uno de estos factores es la motivación.

La motivación es un proceso psicológico con un papel muy importante en la modalidad de educación virtual que puede ser manejado por los mismos estudiantes para las actividades que se proponen y para su propio proceso de aprendizaje, el cual puede llevar a tener el cumplimiento de objetivos con una actitud activa frente a los retos que se le presenten.

Almaguer y Elizondo (2002), citados por Quinche y González (2011), plantean que la motivación es un aspecto que se encuentra presente en todos los procesos de enseñanza y aprendizaje, en los que interactúan esfuerzos orientados a algunas acciones con efectos 
consecuentes para la obtención de resultados, influyendo de manera positiva o negativa en los procesos cognitivos.

Un buen maestro se preocupa por llevar a cabo el mantenimiento de un nivel elevado de motivación en sus estudiantes, planeando y planteando de forma correcta las actividades que se llevarán a cabo en los cursos virtuales. Ello nos lleva a plantearnos una pregunta: ¿Los estudiantes en la modalidad de educación virtual aprenden a manejar por sí mismos, de manera correcta, su propia motivación? (Aebli, 1991).

Debido a que la motivación es un factor intrinseco en el individuo, sobre estos factores sí se puede ejercer un control personal. Como lo menciona Rodríguez (2009) al decir sobre la motivación que "la motivación que origina el mismo sujeto-denominada motivación intrínseca-; está casi siempre relacionada con el deseo personal de satisfacer necesidades, intereses y expectativas y cuyos resultados se esperan disfrutar (p. 89)".

Dentro del marco de la educación virtual encontramos otro factor que tiene mucha importancia y es muy influyente dentro de este proceso: la autonomia. Ésta hace referencia al momento en el que el estudiante toma conciencia de que su proceso de formación académica lo debe asumir él, solo con orientación.

La autonomía juega un papel fundamental dentro del contexto de la educación y la formación de las personas, ya que, como lo mencionan Ferroni y Velásquez (2005), el aprendizaje es un proceso autónomo donde el estudiante hace apropiación de los conocimientos teóricos y la aplicación de los mismos a partir de la toma de conciencia sobre su responsabilidad en cuándo, cómo y cuánto debe aprender, para saber más y mejor sobre un área o un quehacer específico. Se debe llegar a que los estudiantes universitarios sepan claramente cuándo y por qué aprender (Freixes, 2008).

Rosalba Rodriguez (2009) asegura: "los estudiantes aprenden por su propia cuenta y, asi mismo, tienen más responsabilidad y adquieren un cierto grado de autonomía en el aprendizaje (p. 55)". Se requiere de un aprendizaje independiente pero ello no significa aislamiento, sino que sería la representación directa de la autonomía con la orientación de parte de los docentes-tutores.

Los estudiantes dejarán de tener un rol pasivo y asumirán un rol más activo, ya que la educación virtual brinda la oportunidad de desarrollar en ellos un nuevo comportamiento, siendo ellos mismos quienes buscan la información y logran cumplir con los objetivos académicos del curso que se han propuesto (González, 2006). Es importante que el estudiante de educación virtual tenga una gran participación en su propio aprendizaje actuando con proactividad y con autonomia, fijándose metas propias, reflexionando sobre sus propias destrezas y estrategias que aplica y con una participación activa en la comunicación y en el aprendizaje colaborativo (Bautista, Borges \& Fores, 2006), ya que el trabajo grupal de los estudiantes es fundamental para la formación en el sentido de compartir experiencias mediante la adquisición de nuevos saberes (Calle, 2009) y aprendiendo de los demás compañeros (Panckhurts y Marsh, 2011).

De esta manera se observa que los roles de los estudiantes evolucionan y así mismo los roles de los docentes o tutores también se transforman, llevando a que éstos cambien del rol docente ubicado únicamente en el lugar de poseedor del conocimiento y del alumno como simple receptor de éste, en tanto que espera encontrar constantemente el saber en la figura del profesor (Valcarce y Venticinque, 2008), a unos roles en los cuales el estudiante también es poseedor del conocimiento y puede darle sentido a lo que se encuentra en su proceso de enseñanza-aprendizaje mediante una posición más activa en su proceso de formación académica.

Aebli (1991), refiere que para que el aprendizaje autónomo tenga un buen provecho, se deben incluir también algunas habilidades y actitudes adicionales, ancladas profundamente en la personalidad de los tutores, ya que son éstos quienes generarán la interacción con los estudiantes la interacción se convierte en un aspecto central de cualquier experiencia educativa, y más aún cuando se intenta promover el desarrollo del aprendizaje critico, reflexivo y autónomo (Sánchez, 2009).

Garduño (2005) menciona que se debe tener en cuenta que el uso adecuado de los métodos pedagógicos para la enseñanza virtual se deben convertir en un aspecto fundamental en la 
estructura, organización y estilo de la comunicación. La interacción entre tutores, asesores y estudiantes, se debe establecer a través de medios de comunicación que no impliquen la presencialidad ni la sincronía, pero que incidan significativamente en el aprendizaje.

Es precisamente el aprendizaje significativo y no el repetitivo, el que se debe fomentar en cualquier modalidad educativa, a partir de la construcción de significados, aspecto fundamental en los procesos de enseñanza aprendizaje (Carrasco, 1997), involucrando la construcción de modelos mentales (Moreira, Greca, Rodríguez y González, 2002).

Para lograr buenos resultados mediante el aprendizaje significativo, se debe considerar la comprensión de lectura como otro aspecto fundamental en los procesos de enseñanzaaprendizaje en la educación virtual. Rodriguez (2009) plantea al respecto que "en educación virtual, la lectura se constituye en el proceso básico del acto académico, porque es en esta habilidad donde radica la posibilidad de interpretar y comprender el texto escrito (editado o digitalizado)" (pg. 62). La comprensión lectora debe ser una competencia a desarrollar dentro del marco de la educación virtual debido a las nuevas formas en que se organizan y se entregan los contenidos de estudio (Borrego, Rodríguez, Walle \& Ponce, 2008).

Así como debe estar-o desarrollarse- la competencia de interpretación de textos, también se resalta que existen otras competencias adicionales que deben poseer los estudiantes de educación virtual, como lo plantea Padilla (2008) al citar el siguiente aspecto en relación a las competencias individuales y relacionales que deben presentarse en un buen proceso de educación virtual, con el objetivo de orientar a quien desee ahondar en este tema:

Competencias individuales

Motivación

Responsabilidad

Decisión

Iniciativa
Actitud

Liderazgo

Competencias relacionales

Espíritu de equipo y colaboración (sinergia)

Comunicación y relaciones interpersonales

Transmisión y difusión de la información

Capacidad para ordenar y estimular a los demás

Es sabido que el éxito en los procesos de aprendizaje se puede garantizar siempre y cuando se sustente con teorías sobre enseñanza-aprendizaje. De igual manera, cabe mencionar otro aspecto relevante dentro de la formación de los estudiantes en modalidades de educación virtual y es lo referente a ciertas competencias que también deben poseer los docentes o tutores, siendo éstos personas actualizadas en el arte de enseñar y transformar (Abdala, Castiglione e Infante, 2008). Los docentes o tutores de la educación virtual deben tener unas características especiales que los lleven a familiarizarse con el uso y manejo de las TIC y su metodología específica (Guerra, González \& Garcia, 2010).

Además, los docentes o tutores, aunque no tengan una aproximación presencial con sus estudiantes, deben dominar los contenidos de su materia. Si no conocen y dominan unas determinadas, específicas y pertinentes estrategias de enseñanza, dificilmente pueden conseguir el objetivo prioritario de su función de enseñante, tutor, guía o docente: que sus estudiantes aprendan (Sevillano, 1995).

En la actualidad, el concepto de competencias está en furor en lo referente a enseñanza universitaria, con el objetivo de que se desarrollen procesos formativos que doten a los estudiantes de las competencias que llevan a mejorar su preparación para el ejercicio profesional y para la formación a lo largo de su vida. Es decir, se replantea la formación llevada al 
conocimiento para trabajar en una formación orientada al aprendizaje (Zabalza, 2003).

Las metodologías pedagógicas actuales deben propender por llevar a que los estudiantes aprendan no sólo los contenidos sino también el aprendizaje mismo. Deben convertirse en aprendices autónomos y ésta es la apuesta de varias instituciones encargadas de la educación superior en que se insta a llegar a nuevos paradigmas de enseñanza-aprendizaje, centrados en el aprendizaje autónomo a lo largo de la vida de los estudiantes (Álvarez, Rodriguez, SanzAblanedo \& Fernández-Martínez, 2008).

Desde la modalidad de educación virtual, es necesario dejar claro que requiere que los cursos o módulos a desarrollar como cursos virtuales, sean de una alta calidad y que potencien en los estudiantes la posibilidad de desarrollar sus máximas capacidades para interactuar y relacionarse con sus tutores-docentes y compañeros de aula; aprendiendo en un ambiente educativo que se encuentra mediado por las tecnologías de la información y las comunicaciones (Ardila, 2011).

Se tiene que hacer énfasis en la creación de estrategias que logren el objetivo de que los estudiantes aprendan a aprender, pero ¿qué se quiere decir con el proceso de aprender a aprender y cómo se relaciona éste con el aprendizaje autónomo? Aebli (1991), en su libro Factores de la enseñanza que favorecen el aprendizaje autónomo nos da una respuesta aproximada al hacerse la pregunta "¿para qué aprender a aprender? (p. 30)". Y así es como contesta: "aprendemos a aprender para convertirnos en aprendices autónomos. Quien ha aprendido a aprender no necesita ya de alguien que lo guíe en el aprendizaje. Ya es capaz de aprender por sí mismo (...)", pero ¿para qué el aprendizaje autónomo?

Aprendizaje autónomo para aprender más: se sabe que en la medida en que los estudiantes trabajen de manera independiente, aprendan y experimenten más allá de lo que los contenidos nos transmiten directamente, el proceso de aprendizaje puede tener un efecto más favorable en ellos.

Aprendizaje autónomo como preparación para el trabajo: en la actualidad, la vida laboral, con su presión innovadora, los cambios tecnológicos y de mercado, exige una adaptación permanente por parte de los trabajadores.
Brocket y Hiemtra (1993), desarrollan el concepto de aprendizaje autodirigido en lugar de aprendizaje autónomo. En relación a ello afirman que "la autodirección en el aprendizaje es una combinación de fuerzas tanto interiores como exteriores de la personas que subrayan la aceptación por parte del estudiante de una responsabilidad cada vez mayor respecto a las decisiones asociadas al proceso de aprendizaje" (p. 15), definición similar a la planteada por Zimmerman (1989) citado por Góngora (s.f.), al referirse a la autogestión del aprendizaje.

Quien se encuentra en situación de aprendizaje, debe lograr inicialmente algunos rendimientos que implican competencia social y la motivación correspondiente. Se considera por tanto que también se prepara a una persona para el aprendizaje autónomo en la media en que se le capacita y motiva a tomar parte y trabajar de manera responsable consigo mismo (Aebli, 1991).

El objetivo central del aprendizaje autónomo se encuentra en el marco de lograr que los estudiantes aprendan a aprender, lo cual conduce a la autonomía en el aprendizaje, siendo indispensable enseñar a los estudiantes a que adopten o incorporen progresivamente estrategias de aprendizaje, enseñarles a ser más conscientes sobre la forma como aprenden y así puedan enfrentar de manera satisfactoria diferentes situaciones de aprendizaje (Manrique, 2004).

Sin embargo, es necesario resaltar de nuevo el hecho de que el estudiante en modalidades de enseñanza virtuales, es altamente activo en su proceso de aprendizaje, por tanto tiene que ser un estudiante con un alto sentido crítico y reflexivo, ya que la simple transmisión de saberes que no lleven a una actitud de discusión reflexiva, no favorece la implicación del estudiante en la situación de enseñanza y aprendizaje (Del Río, Venticique, Valcarce \& Diez, 2008). "La actitud critica es el único medio por el cual el hombre realizará su vocación natural de integrarse, superando la actitud del simple ajuste o acomodamiento" (Freire, 2005b, citado por Pérez, 2009) (p. 12).

Manrique (2004), a partir de su revisión teórica, encuentra algunas estrategias necesarias para el desarrollo del aprendizaje autónomo en modalidades de educación virtual. A continuación se refieren tales estrategias: 


\section{Desarrollo de estrategias afectivo-motivacionales.}

Tales estrategias se orientan a que los estudiantes sean conscientes de las capacidades y estilos que ellos mismo tienen para aprender. También se orientan a que éstos desarrollen autoconfianza en sus capacidades y habilidades, logren una motivación intrinseca hacia todas las actividades de aprendizaje y puedan superar las dificultades que se presentan. Estas estrategias fortalecen en el estudiante la voluntad, el querer aprender (Alonso y López 1999, citados por Manrique, 2004), $\mathrm{y}$ les ayudan a fortalecer modelos mentales (ideas, creencias, convicciones) positivos sobre sí mismos y sus capacidades de aprender. Si los estudiantes no están familiarizados con esta forma de enseñanza y aprendizaje, tales estrategias son fundamentales para poder fortalecer las actitudes hacia el aprendizaje autónomo.

\section{Desarrollo de estrategias de autoplanificación}

Estas estrategias están relacionadas con diferentes aspectos cuyos propósitos se encuentran encaminados en última instancia a lograr la formulación de un plan de estudio realista y efectivo. Tal plan es el que permitirá a los estudiantes conocer los aspectos relacionados con las tareas y las condiciones en que se deben realizar.

Identificación de metas de aprendizaje propuestas para ser asumidas y reorientadas con el objetivo que adquieran significación para los estudiantes. Pueden presentarse a nivel individual o cooperativas.

Identificación de condiciones físicas y ambientales para el estudio (tiempo que se dispone, horarios de estudios, recursos o materiales con los que se cuentan y variables ambientales).

Análisis de las condiciones de las tareas asignadas: evaluar la complejidad de las actividades, secuencia a seguir, tipo de actividad, condiciones esperadas como resultado.

Selección de las estrategias más convenientes para abordar los temas y la forma de estudio, basado en las condiciones anteriormente señaladas y las metas propuestas. Tales estrategias hacen referencia a la manera como se deben enfrentar la lectura, el análisis e interpretación de la información, manejo de las tecnologias de la información y la comunicación, habilidades de comunicación e interacción para un aprendizaje colaborativo.

\section{Desarrollo de estrategias de autorregulación}

Estas estrategias conducen a la aplicación de estrategias seleccionadas para el estudio y el aprendizaje, revisión continua de sus avances, dificultades y éxitos en sus tareas según las metas de aprendizaje que se hayan trazado. En estas estrategias se incluyen la generación de alternativas de solución y previsión de consecuencias, la toma de decisiones oportunas de acciones a realizar o condiciones que deban ser modificadas para lograr sus propósitos.

\section{Desarrollo de estrategias de autoevaluación}

Tales estrategias se orientan a la evaluación de los estudiantes, de las tareas o actividades realizadas y de las estrategias utilizadas. Los estudiantes pueden comparar información que van obteniendo y valoran la efectividad que ha tenido su planificación llevada a cabo y la manera como participaron en sus respectivos cursos. También evalúan el nivel logrado en las metas de aprendizaje.

\section{Conclusiones}

Teniendo en cuenta lo mencionado a lo largo de este artículo, son varias las conclusiones que se podrian presentar. Sin embargo lo que más se puede resaltar es que la educación virtual es una modalidad que cuenta con las herramientas necesarias para que pueda favorecer la autonomía en los procesos de aprendizaje de las personas. No se trata de establecer una yuxtaposición de lo virtual sobre lo presencial, sino de reconocer que es posible la transformación de la educación tradicional a la educación apoyada por la tecnología (Edel-Navarro, 2010), y que ésta se puede convertir en una gran herramienta pedagógica para favorecer el desarrollo del aprendizaje autónomo. 
Como lo menciona Rúe (2010), frente a "la especial insistencia en el aprendizaje autónomo del alumno, no conviene entenderlo como una respuesta puntual frente a un determinado tipo de actividades, sino que constituye una competencia que los alumnos deben saber y adquirir y los docentes deben saber cómo transmitir" (p. 7).

Es indiscutible que los últimos años se ha observado un gran interés por la educación virtual, por medio de investigaciones, debates y controversias alrededor de las ventajas y limitaciones que ésta ofrece en los procesos relacionados con el aprendizaje autónomo. Cada día es mayor el número de personas convencidas de estas formas de aprendizaje; sin embargo es necesario reconocer que hay quienes todavía son escépticos sobre los resultados y el grado de generalización de las prácticas autónomas. 


\section{Referencias}

1. Abdala, S., Castiglione, A. e Infante, L. (2008). La educación universitaria en el nuevo contexto del mercado laboral: entre la lógica de las demandas y la identidad Institucional. Cuadernos FHyCS UNju, número 34, 41-51.

2. Aebli, H. (1991). Factores de la enseñanza que favorecen el aprendizaje autónomo. Madrid: Narcea.

3. Álvarez, F., Rodríguez, J., Sanz-Ablanedo, E. \& Fernández-Martínez, M. (2008). Aprender enseñando: Elaboración de materiales didácticos que facilitan el aprendizaje autónomo. Formación Universitaria, 6 (1), 19 - 28.

4. Ardila, M. (2011). Indicadores de calidad de las plataformas educativas digitales. Educación, 1 (14), $189-206$.

5. Badía, A. y Monimó, J. (2001). La Incógnita de la educación a distancia. Editorial S.I.

6. Badía, A. (2001). Ser estratégico y autónomo aprendiendo: Unidades didácticas de enseñanza estratégica. Grao.

7. Batista, E. (s.f.). Lineamientos pedagógicos para la enseñanza y el aprendizaje. Bogotá: Univ. Cooperativa de Colombia.

8. Bautista, G., Borges, F. y Fores, A. (2006). Didáctica Universitaria en entornos virtuales de enseñanza-aprendizaje. Madrid: Nercea S.A.

9. Borrego, N., Rodríguez, H., Walle, R. \& Ponce, J. (2008). Educación superior virtual en América Latina: Perspectiva tecnológicaempresarial. Formación Universitaria, 5 (1), 3-14.

10. Brocket, R. y Hiemtra, R. (1993). El aprendizaje autodirigido en la educación de adultos. Barcelona: Paidós.

11. Calle, M. (2009). La tutoría como mediación para el desarrollo autónomo del estudiante. Revista Tabula Rasa, número 11, 309-328
12. Carrasco, J. (1997). Hacia una enseñanza eficaz. Madrid: Ediciones Rialp S.A.

13. Del Río, M., Venticinque, N., Valcarce \& Díez, B. (2008). Las condiciones para un aprendizaje universitario más autónomo desde la mirada del alumno. Anuario de Investigaciones, volúmen XV.

14. Edel-Navarro, R. (2010). Entornos virtuales de aprendizaje. Revista Mexicana de Investigación Educativa, 44 (15), 7-15.

15. Enríquez, A. (2001). Diagnóstico de la educación superior a distancia. México: Asociación Nacional de Universidades e Instituciones de Educación Superior, AUNIES.

16. Facundo, A. (2004). La virtualización en la perspectiva de la modernización de la educación superior: Consideraciones pedagógicas. Poliantea. Revista académica y cultural Fundación Politécnico Grancolombiano Institución Universitaria, número 2, 8-25.

17. Fernández, A. y Córdoba, D. (2006). Nuevos ambientes de aprendizaje en postgrado: Integrando conocimientos, estrategias y herramientas tecnológicas. Revista Investigación y Postgrado, 1 (21), 221-230.

18. Ferroni, E. y Velásquez, H. (2005). Educación a distancia para el salto académico (Parte I). Poliantea. Revista académica y cultural Fundación Politécnico Grancolombiano Institución Universitaria, número 4. Julio Diciembre, 7-34.

19. Freixes, N. (2009). Cómo mejorar la docencia universitaria: el punto de vista del estudiantado. Revista Complutense de Educación, 1 (20), 117-134.

20. Garduño, R. (2005). Enseñanza virtual sobre la organización de recursos informativos digitales. UNAM.

21. Garduño, R. (2009). Contenido educativo en el aprendizaje virtual. Investigación Bibliotecológica, 47 (23), 15-44. 
22. Góngora, J. (s.f.). Módulo Curso de Sistemas de Aprendizaje para un Aprendizaje autónomo. Curso, pedagogías del aprendizaje significativo de la especialización en pedagogía para el desarrollo del aprendizaje autónomo.

23. González, S. (2006). La universidad entre lo presencial y lo virtual. México: UAEM.

24. Gorbaneff, Y. y Cancino, A. (2009). Mapa conceptual para el aprendizaje basado en problemas. Revista de estudios gerenciales, 10 (25), $111-124$.

25. Guerra, S., Gonzáles, N. \& García, R. (2010). Utilización de las TIc por el profesorado universitario como recurso didáctico. Revista Científica de Educomunicación, 35 (XVIII), 141-148.

26. Gutiérrez, C., Hernández, L., González, G., Guevara, C. \& Flórez, J. (2004). La enseñanza virtual en el contexto de la educación actual. Revista Ciencias Salud. (Jul - Dic).

27. Imbernón, F., Silva, P. \& Guzmán, C. (2011). Competencias en los procesos de enseñanzaaprendizaje virtual y semipresencial. Revista Científica de Educomunicación, 36 (XVIII), 107-114.

28. Iriarte, A. (2010). Hacia el desarrollo de la educación superior virtual. Revista Mexicana de Investigación educativa, 44 (15), 185-189.

29. López, M. (2005). Una Comunidad virtual como herramienta de difusión de la educación a distancia en una Institución de Educación Superior tradicional venezolana. Rev. Ped. [online]. sep. 2005, vol.26, no.77 [citado 03 mayo 2011], p.2-6. Disponible en la World Wide Web: <http://www.scielo.org.ve/ scielo.php?script=sci_arttext\&pid=S079897922005000300002\&lng=es\&nrm $=$ iso $>$.

30. Manrique, L. (2004). El aprendizaje autónomo en la educación a distancia. Primer congreso virtual Latinoamericano de educación a distancia. Latineduca2004.com Departamento de educación, Pontificia Universidad Católica del Perú. Perú.
31. Merino, J. (2009). La escuela centrada en la comunidad: Un modelo de escuela inclusiva para el Siglo XXI. Revista Complutense de Educación, 1 (20), 33-52.

32. Monguet, J., Fábregas, J. Delgado, D., Grimón, F. \& Herrera, M. (2006). Efecto del Blendend Learning sobre el rendimiento y la motivación de los estudiantes. Revista Inerciencia, 3 (31), 190.

33. Moreira, M., Greca, I., Rodríguez, M. \& González, A. (2002). Mental Models and Conceptual Models in the Teaching and Learning of Science. Conferencia dictada en el XX Encuentro de didáctica de las Ciencias Experimentales. Tenerife.

34. Padilla, J. (2008). Globalización y educación superior: un reto en la formación del docente universitario. Itinerario educativo, número 4.

35. Páez, H. y Arreaza, E. (2005). Uso de una Plataforma virtual de aprendizaje en educación superior. Caso nicenet.org. Maracay.

36. Panckhurst, R. y Marsh, D. (2011). Utilización de redes sociales para la práctica pedagógica en la enseñanza superior impartida en Francia: perspectivas del educador y del estudiante. En: "El impacto de las redes sociales en la enseñanza y el aprendizaje" [monográfico en línea]. Revista de Universidad y Sociedad del Conocimiento (RUSC). Vol. 8, n.1, págs. 233-252. UOC.).

37. Pérez, A. (2009). El desarrollo de la lectura crítica: una vía hacia la alfabetización mediática en Educación Superior. Letras. [online]. ene. 2009, vol.51, no.78 [citado 03 Octubre 2011], p.309-356. Disponible en : <http:// www.scielo.org.ve/scielo.php?script=sci arttext\&pid=S0459-12832009000100010\&Ing=e $\mathrm{s} \& \mathrm{nrm}=\mathrm{iso}>$.

38. Pirela De Odón, D. (2004). Ideas para la formulación de políticas universitarias referidas al uso de tecnologías de información y comunicación y la educación virtual. Rev. Ped. [online]. Ene. 2004, vol.25, no.72 [citado 3 de mayo 2011], p.149-170. Disponible en la World Wide Web: <http://www.scielo.org. ve/scielo.php?script=sci_arttext\&pid=S0798- 
$97922004000100006 \& \operatorname{lng}=e s \& n r m=i s o>$. ISSN 0798-9792.

39. Quinche, J. y González, F. (2011). Entornos virtuales 3D, alternativa pedagógica para el fomento del aprendizaje colaborativo y gestión del conocimiento en Uniminuto. Formación Universitaria, 2 (4), 45-54.

40. Rada, D. (2006). Participación de los docentes en el foro virtual: usos pedagógicos de los recursos tecnológicos. Revista Educere, 34 (10), 443-453.

41. Real Academia de la Lengua. (2001). Diccionario de la lengua Española. 22ª Edición. España.

42. Rincón, D. y Romero, M. (2006). Ciencia, tecnología y educación en Venezuela: perspectiva de una sociedad emergente. Revista de Ciencias Sociales, 1 (XII), 72-83.

43. Rodríguez, R. (2009). Metodología del trabajo académico. Curso SIUP de la especialización en pedagogía para el desarrollo del aprendizaje autónomo.

44. Rué, J. (2009). El cambio en la Universidad, sus epistemologías y consecuencias de las mismas. Revista Complutense de Educación, 2 (20), 295-317.

45. Rué, J. (2010). El aprendizaje autónomo en Educación Superior. Revista Complutense de Educación, 1 (21), 211-223.

46. Sánchez, A. (2009). Nuevos modos de interacción educativa: análisis lingüístico de un foro virtual. Educación y Educadores, 2 (12).

47. Serrano, J. y Narváez, P. (2010). Uso de Software libre para el desarrollo de contenidos educativos. Formación Universitaria, 6 (3).

48. Sevillano, M. (1995). Estrategias de enseñanza y aprendizaje con medios y tecnología. Madrid: Ed. Ramón Areces.
49. Taylor, S. y Bogdam, R. (1996). Introducción a los métodos cualitativos de investigación. La búsqueda de significados. Barcelona: Paidós.

50. Torres, V. y Benítez, R. (s.f.). Programa en línea para la formación de docentes en el marco de la Reforma Integral de la Educación Media Superior en México. Issue. 4, Vol. 7. Universidad Autónoma de Nayarit. México.

51. Valcarce, M. y Venticinque, N. (2008). La evolución del perfil docente en un dispositivo de autoformación. Anuario de investigaciones, volúmen XIV.

52. Varini, R. y Axt, M. (2008). Para uma posiçao enunciativa no discurso pedagógico mediado por ambientes virtuais de aprendizagem. Interface. Comunicaçao Saude Educaçao, 25 (12), 347-361.

53. Zabalza, M. (2003). Competencias docentes del profesorado universitario: calidad y desarrollo profesional. 


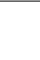

